\section{An Interesting Image}

\author{
Chitra Mehta $^{1}$ Yatin Mehta ${ }^{1}$ \\ ${ }^{1}$ Department of Critical Care, Medanta The Medicity, Gurgaon, \\ Haryana, India
}

J Card Crit Care TSS 2018;2:48

Pneumopericardium is accumulation of air in the pericardium. It is less frequent than pneumomediastinum and pneumothorax. ${ }^{1}$ It develops secondary to several reasons. Infection generally associated with pneumopericardium is Mycobacterium tuberculosis; however, there are a handful of reports of fungal infections. Review of literature shows Histoplasma, Aspergillus, and Candida to be most commonly associated with fungal pneumopericardium. Mucormycosis resulting in pneumopericardium has been reported only once. ${ }^{2}$

A 53-year-old man presented with 4 weeks history of generalized weakness, intermittent fever, cough, and vague chest pain. He had notable history of long-standing diabetes, hypertension, and chronic kidney disease. He received a deceased donor renal transplant 4 months prior to current admission. He was receiving maintenance immunosuppression therapy with steroids, tacrolimus, and mycophenolate mofetil with valganciclovir and trimethoprim-sulfamethoxazole for microbial prophylaxis. His chest roentgenogram showed bilateral cavitating pneumonia. Transthoracic two-dimensional (2D) echocardiogram revealed normal ejection fraction with mild pericardial effusion. He needed ventilatory support within a day of admission. Respiratory specimens obtained bronchoscopically were negative for acid-fast bacilli and fungus on staining. Mycobacterial GeneXpert was also negative. Bronchial aspirate aerobic culture grew Acinetobacter baumannii sensitive to colistin. The patient was put on meropenem, liposomal amphotericin B, clindamycin, and trimethoprim-sulfamethoxazole initially, and later polymyxin B was added as per the culture. A computed tomography (CT) of the head performed for drowsy state showed right frontal lobe abscess. The patient underwent craniotomy and abscess drainage on seventh day of admission. Hyaline aseptate fungal hyphae were demonstrated on $\mathrm{KOH}$ staining of abscess fluid, which were identified as Mucor. Posaconazole was also added. On the 12th day of admission, the patient was found to have pneumopericardium on bedside chest radiograph ( - Fig. 1). Contrast-enhanced CT of the chest showed a large right lower lobe cavity possibly breaching the pericardium with resultant pneumopericardium ( - Fig. 2). Despite being on optimal antifungal therapy, the patient succumbed to disseminated mucormycosis.

The purpose of this letter is to highlight the noteworthy association of mucormycosis with pneumopericardium.
Address for correspondence Chitra Mehta, MBBS, DNB (Respiratory Medicine), FNB (Critical Care Medicine), Department of Critical Care, Medanta The Medicity, Gurgaon 122001, Haryana, India (e-mail: mehtachitra@hotmail.com).

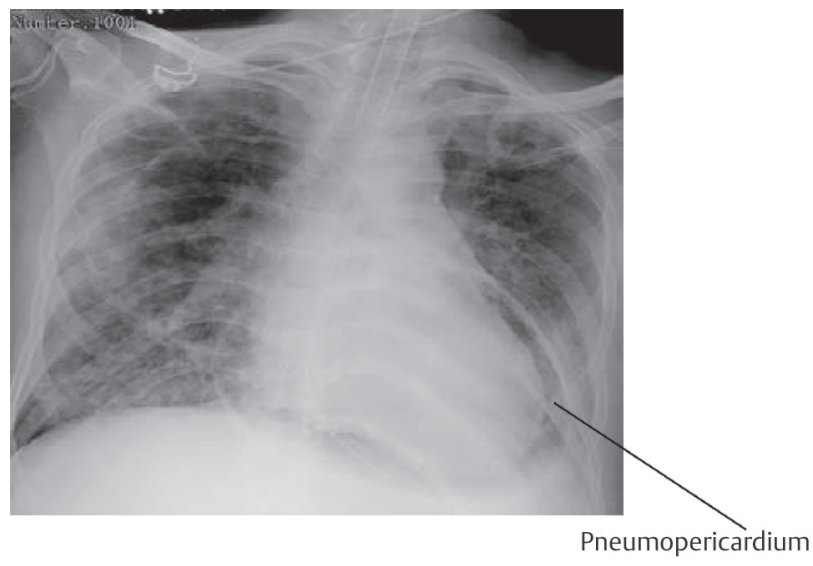

Fig. 1 Pneumopericardium on chest X-ray.

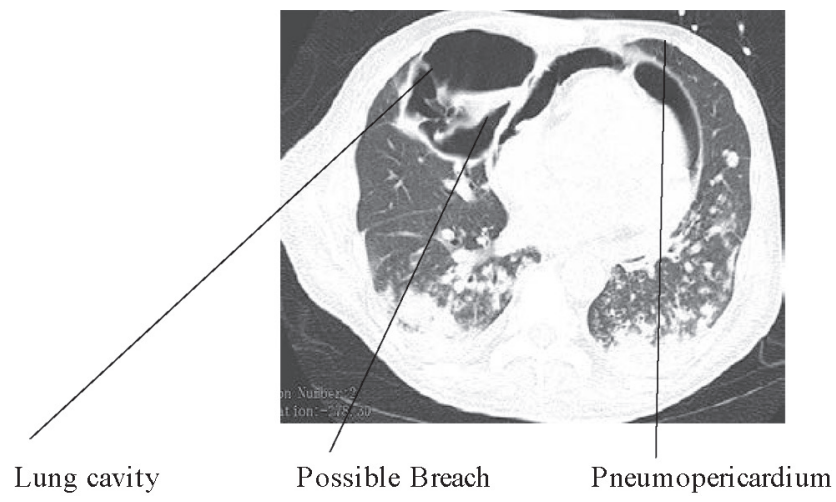

Fig. 2 CT of the chest showing lung cavity and pneumopericardium.

\section{Conflict of Interest}

None.

\section{References}

1 Celik T, Iyisoy A, Kursaklioglu H, Gunay C, Yuksel UC, Isik E. A case of pneumopericardium following endomyocardial biopsy. J Card Surg 2007;22(6):519-521

2 Khan S, Waqar Elahi M, Ullah W, et al. Invasive mucormycosis induced pneumopericardium: a rare cause of pneumopericardium in an immunocompromised patient. Case Rep Infect Dis 2017;2017:1424618
License terms

(우 (1) $\odot \circledast$ 